\title{
Computation of the Structured Singular Value via Moment LMI Relaxations
}

\author{
Dario Piga*,
}

\begin{abstract}
The Structured Singular Value (SSV) provides a powerful tool to test robust stability and performance of feedback systems subject to structured uncertainties. Unfortunately, computing the SSV is an NP-hard problem, and the polynomial-time algorithms available in the literature are only able to provide, except for some special cases, upper and lower bounds on the exact value of the SSV. In this work, we present a new algorithm to compute an upper bound on the SSV in case of mixed real/complex uncertainties. The underlying idea of the developed approach is to formulate the SSV computation as a (nonconvex) polynomial optimization problem, which is relaxed into a sequence of convex optimization problems through moment-based relaxation techniques. Two heuristics to compute a lower bound on the SSV are also discussed. The analyzed numerical examples show that the developed approach provides tighter bounds than the ones computed by the algorithms implemented in the Robust Control Toolbox in Matlab, and it provides, in most of the cases, coincident lower and upper bounds on the structured singular value.
\end{abstract}

Index Terms-Structured Singular Value, Robust Control, Polynomial optimization, Moment-based LMI relaxations.

\section{INTRODUCTION}

The Structured Singular Value (SSV), introduced by Doyle in [1], provides a powerful tool to test robust stability and performance of closed-loop Linear Time-Invariant (LTI) systems subject to structured uncertainties. Unfortunately, computing the SSV is an NP-hard problem [2], [3] and thus, for medium/large-scale problems commonly encountered in industrial applications, the exact value of the SSV cannot be calculated within an acceptable amount of computational time. In order to overcome this issue, polynomial-time algorithms have been developed to compute lower and upper bounds on the SSV [4]-[13] in case of real, complex or mixed real/complex uncertainties. Upper bounds provide sufficient (and thus conservative) conditions to guarantee robust stability of feedback systems under a specified level of uncertainty. The conservativeness of the upper bounds can be evaluated by computing lower bounds on the SSV, which, besides providing sufficient conditions for instability, also give the values of structured uncertainties destabilizing the closed-loop system.

Although most of the previously cited algorithms were developed more than twenty years ago (and some of them are also implemented in the wide-spread commercial Matlab toolbox Robust Control Toolbox [14]), it is still worth developing new algorithms to compute tighter bounds on the $\mathrm{SSV}$, in order to reduce the conservativeness in analysing the robust properties of feedback systems, and thus pushing the

* The author is with the IMT Institute for Advanced Studies Lucca, Piazza San Francesco 19, 55100 Lucca, Italy. email:dario.piga@imtlucca.it performance limits of the controlled systems. In this paper, a novel approach is proposed to compute an upper bound on the SSV through convex optimization. The computation of lower bounds is also discussed. No limitations on the considered perturbations are assumed, in the sense that the proposed algorithm is able to deal with pure real, pure complex and mixed real/complex uncertainties. The main idea of the discussed procedure is to formulate the calculation of the SSV as a nonconvex optimization problem with Linear Matrix Inequality (LMI) and bilinear constraints. An approximation of the solution of the formulated optimization problem is then computed through moment-based LMI relaxations, originally proposed in [15] and [16] to relax polynomial optimization problems into a sequence of convex Semidefinite Programming (SDP) problems. It has been observed that, in practice, moment LMI relaxations provide an accurate (and sometimes exact) approximation of the global optimum of a generic polynomial optimization problem (see, e.g., [17]). Furthermore, conditions for finite convergence of the moment-based relaxations are also available [18]. The algorithm developed in this paper for the computation the SSV benefits from the mentioned advantages of the moment-based relaxations. In most of the examples analyzed by the author (some of them reported in Section VI), the computed lower and upper bounds coincide, thus yielding the exact value of the SSV.

The paper is organized as follows: preliminary definitions and notations used in the paper are first introduced in Section II. In Section III, the computation of the SSV is formulated as a polynomial optimization problem, and the computation of an upper bound on the SSV is discussed in Section IV. In Section V, two heuristics to compute a lower bound on the SSV are presented. Two numerical examples are reported in Section VI to show the performance of the developed algorithm. A comparison with the results obtained by the Robust Control Toolbox is also provided.

\section{NOTATION AND PRELIMINARIES}

The following notation will be used throughout the paper. $N^{\mathrm{H}} \quad$ complex conjugate transpose of the matrix $N$.

$\otimes \quad$ Kronecker product.

$\operatorname{diag}(x)$ diagonal matrix, whose diagonal entries are the components of the vector $x$.

$x_{i} \quad i$-th component of the vector $x$.

$\mathbb{N}_{0}^{n} \quad$ set of $n$-dimensional vectors with nonnegative integer components.

$\mathcal{A}_{h}^{n} \quad$ set defined as $\left\{\alpha \in \mathbb{N}_{0}^{n}: \sum_{i=1}^{n} \alpha_{i} \leq h\right\}$. 
$Z^{\alpha} \quad$ shorthand notation for $Z_{1}^{\alpha_{1}} Z_{2}^{\alpha_{2}} \cdots Z_{n}^{\alpha_{n}}=\prod_{i=1}^{n} Z_{i}^{\alpha_{i}}$.

$\mathbb{R}_{h}[Z] \quad$ set of real-valued polynomials in the indeterminant $Z \in \mathbb{R}^{n}$ with degree less or equal than $h$.

$b_{h}(Z) \quad$ canonical basis of $\mathbb{R}_{h}[Z]$, i.e., $b_{h}(Z)=\left\{Z^{\alpha}\right\}_{\alpha \in \mathcal{A}_{h}^{n}}$.

$\left\{g_{\alpha}\right\}_{\alpha \in \mathcal{A}_{h}^{n}}$ set of coefficients of the polynomial $g \in \mathbb{R}_{h}[Z]$

in the canonical basis $b_{h}(Z)$, i.e., $g(Z)=\sum_{\alpha \in \mathcal{A}_{h}^{n}} g_{\alpha} Z^{\alpha}$.

The structured singular value is now formally defined. Let us consider the set of complex matrices $\Delta \subset \mathbb{C}^{s_{1}, s_{2}}$, whose elements $\Delta \in \Delta$ admit the structure:

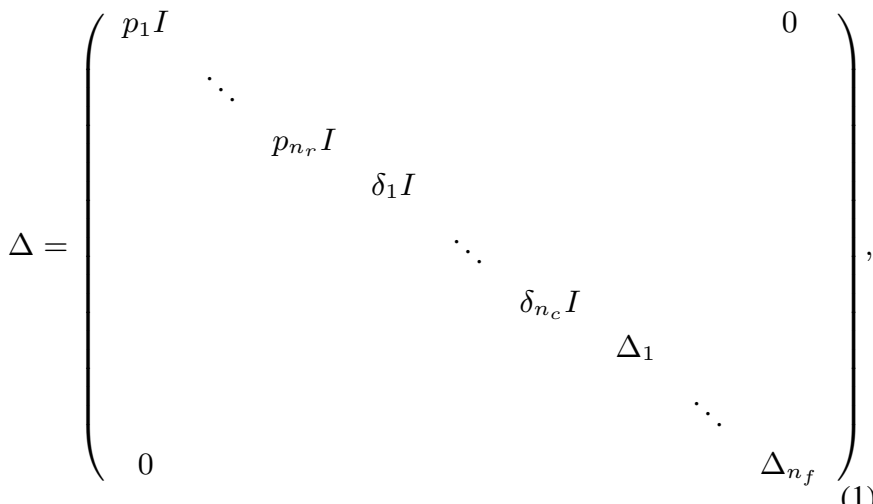

where: $p_{j} \in \mathbb{R}$ (with $j=1, \ldots, n_{r}$ ); $\delta_{j} \in \mathbb{C}$ (with $j=$ $1, \ldots, n_{c}$ ) and $\Delta_{j} \in \mathbb{C}^{p_{j}, q_{j}}$ (with $j=1, \ldots, n_{f}$ ). The sizes of the identity matrices in (1) can be different for different blocks. For a given nonnegative real number $r$, let $\mathcal{B}_{\Delta}^{r}$ be the (open) set of complex matrices $\Delta$ whose admit the structure in (1) and such that $\|\Delta\|<r$, i.e.,

$$
\mathcal{B}_{\Delta}^{r}=\{\Delta \in \Delta:\|\Delta\|<r\} .
$$

Definition 1: Given a complex matrix $M \in \mathbb{C}^{s_{2}, s_{1}}$, the structured singular value of $M$ w.r.t. the uncertainty set $\Delta$ is defined as:

with

$$
\mu_{\Delta}(M)=\frac{1}{r^{*}},
$$

$$
r^{*}=\sup \left\{r \in \mathbb{R} \mid \operatorname{det}(I-M \Delta) \neq 0 \text { for all } \Delta \in \mathcal{B}_{\Delta}^{r}\right\} .
$$

As pointed out in [3], the computation of the SSV $\mu_{\Delta}(M)$ is an NP-hard problem and it requires to verify the nonsingularity of the matrix $I-M \Delta$ against all structured uncertainties $\Delta$ belonging to $\mathcal{B}_{\Delta}^{r}$. In the next section, the problem of checking robust non-singularity of the matrix $I-M \Delta$ will be formulated as a (nonconvex) optimization problem with LMI and bilinear equality constraints, and it will be solved through efficient convex-relaxation techniques.

\section{CHECKING ROBUST NON-SINGULARITY OF THE MATRIX $I-M \Delta$}

First, let us rewrite the constraint $\|\Delta\|<r$ as:

$$
\left(\begin{array}{cc}
r^{2} I & \Delta \\
\Delta^{\mathrm{H}} & I
\end{array}\right) \succ 0 \text {. }
$$

The following theorem (originally presented by the author and coworkers in [19]), provides necessary and sufficient conditions to test robust non-singularity of $I-M \Delta$ against the set of structured uncertainties $\mathcal{B}_{\Delta}^{r}$.
Theorem 1: For a given real number $r \geq 0$, the matrix $I-M \Delta$ is nonsingular for all (structured) uncertainties $\Delta \in$ $\mathcal{B}_{\Delta}^{r}$ if and only if the solution of the following optimization problem is bounded:

$$
\begin{array}{ll} 
& \max _{\substack{x \in \mathbb{C}^{s_{2}} \\
\Delta \in \Delta}}\|x\|_{2}^{2} \\
\text { s.t. } & (I-M \Delta) x=0, \quad\left(\begin{array}{cc}
r^{2} I & \Delta \\
\Delta^{\mathrm{H}} & I
\end{array}\right) \succ 0 .
\end{array}
$$

Proof: First, the "only if" part is proven. If $I-M \Delta$ is robustly nonsingular against the set of (structured) uncertainties $\mathcal{B}_{\Delta}^{r}$, then, for all $\Delta \in \mathcal{B}_{\Delta}^{r}$, only the trivial solution $x=0$ satisfies the constraint $(I-M \Delta) x=0$. Therefore, the solution of Problem (4) is equal to zero, thus bounded. The "if" part is proven by contradiction. Assume that $I-M \Delta$ is not robustly nonsingular against $\mathcal{B}_{\Delta}^{r}$. This means that there exists a matrix $\Delta$ in $\mathcal{B}_{\Delta}^{r}$ such that the solution $x$ of the linear system $(I-M \Delta) x=0$ is not the trivial one, i.e., $(I-M \Delta) x^{*}=0$ for some $x^{*} \neq 0$. Therefore, for every $\beta \in \mathbb{C}$, also $x=\beta x^{*}$ satisfies the constraint $(I-M \Delta) x=0$, and thus $x=\beta x^{*}$ also belongs to the set of feasibility of Problem (4) for any $\beta \in \mathbb{C}$. Then, the solution to problem (4) is unbounded, contradicting the hypothesis.

Corollary 2: The SSV of $M$ w.r.t. $\Delta$ is equal to:

$$
\mu_{\Delta}(M)=\frac{1}{\sqrt{t^{*}}}
$$

where $t^{*}$ is the solution of the following optimization problem:

$$
\begin{aligned}
t^{*}= & \min _{t \in \mathbb{R}} t \\
& x \in \mathbb{C}^{s_{2}} \\
& \Delta \in \boldsymbol{\Delta} \\
\text { s.t. } & t \geq 0, \quad\|x\|_{2}^{2} \geq \bar{x}, \\
& (I-M \Delta) x=0, \quad\left(\begin{array}{cc}
t I & \Delta \\
\Delta^{\mathrm{H}} & I
\end{array}\right) \succeq 0 .
\end{aligned}
$$

with $\bar{x}$ being an arbitrary strictly positive real constant.

Proof: Statement of the Corollary follows directly from the definition of $\mu_{\Delta}(M)$ and from Theorem 1. In fact, for any point $\left(t^{\prime}, x^{\prime}, \Delta^{\prime}\right)$ belonging to the set of feasibility of Problem (6), the solution $x^{\prime}$ of the linear system $\left(I-M \Delta^{\prime}\right) x^{\prime}=0$ is not the trivial one (since $\left\|x^{\prime}\right\|_{2}^{2} \geq \bar{x}>0$ ). Thus, the matrix $\Delta^{\prime}$, which belongs to the set $\mathcal{B}_{\Delta}^{\sqrt{t^{\prime}}} \cup \partial \mathcal{B}_{\Delta}^{\sqrt{t^{\prime}}}$ (with $\partial \mathcal{B}_{\Delta}^{\sqrt{t^{\prime}}}$ being the closure of $\mathcal{B}_{\Delta}^{\sqrt{t^{\prime}}}$ ), makes $I-M \Delta^{\prime}$ singular. Then, $I-$ $M \Delta$ is not robustly nonsingular against the set $\mathcal{B}_{\Delta}^{\sqrt{t^{\prime}}} \cup \partial \mathcal{B}_{\Delta}^{\sqrt{t^{\prime}}}$. The minimum value of $t^{\prime}$ such that $I-M \Delta$ is not robustly nonsingular against the set $\mathcal{B}_{\Delta}^{\sqrt{t^{\prime}}} \cup \partial \mathcal{B}_{\Delta}^{\sqrt{t^{\prime}}}$ is then $t^{*}$. Therefore, for any $t \leq t^{*}$, there is no matrix $\Delta$ belonging to the (open) set $\mathcal{B}_{\Delta}^{\sqrt{t}}$ which makes $I-M \Delta$ singular.

Note that, since both $x$ and $\Delta$ might be complex, Problem (6) involves complex optimization variables. By reminding that an Hermitian matrix $H=H_{\mathrm{re}}+i H_{\mathrm{im}}$ is positive semidefinite if and only if

$$
\left(\begin{array}{cc}
H_{\mathrm{re}} & -H_{\mathrm{im}} \\
H_{\mathrm{im}} & H_{\mathrm{re}}
\end{array}\right) \succeq 0,
$$

Problem (6) can be equivalently rewritten as the following optimization problem with real decision variables: 


$$
\begin{aligned}
& t^{*}= \min _{t, x_{\mathrm{re}}, x_{\mathrm{im}},} t \\
& \Delta_{\mathrm{re}}, \Delta_{\mathrm{im}} \\
& \text { s.t. } t \geq 0, \\
&\left\|x_{\mathrm{re}}\right\|_{2}^{2}+\left\|x_{\mathrm{im}}\right\|_{2}^{2}-\bar{x} \geq 0, \\
&\left(I-M_{\mathrm{re}} \Delta_{\mathrm{re}}+M_{\mathrm{im}} \Delta_{\mathrm{im}}\right) x_{\mathrm{re}}+\left(M_{\mathrm{im}} \Delta_{\mathrm{re}}+M_{\mathrm{re}} \Delta_{\mathrm{im}}\right) x_{\mathrm{im}}=0, \\
&\left(I-M_{\mathrm{re}} \Delta_{\mathrm{re}}+M_{\mathrm{im}} \Delta_{\mathrm{im}}\right) x_{\mathrm{im}}-\left(M_{\mathrm{im}} \Delta_{\mathrm{re}}+M_{\mathrm{re}} \Delta_{\mathrm{im}}\right) \\
& x_{\mathrm{re}}=0, \\
&(8 \mathrm{~d})
\end{aligned}
$$

The computation of $\mu_{\boldsymbol{\Delta}}(M)$ has been then recast into the (nonconvex) polynomial optimization Problem (8) with: a (convex) linear constraint (8b); a (convex) LMI constraint (8f); a (nonconvex) polynomial inequality constraint (8c) and a set of (nonconvex) bilinear equality constraints (8d)-(8e), involving the product among the decision variables $x_{\mathrm{re}}, x_{\mathrm{im}}$, $\Delta_{\mathrm{re}}$ and $\Delta_{\mathrm{im}}$. In the recent years, efficient methods have been proposed in the literature to relax optimization problems with matrix and polynomial constraints into (convex) semidefinite programming problems. Such LMI-relaxation techniques are discussed in [15], [16], [20], [21] and they are based on the problem of moments and on the dual representation of nonnegative polynomials as sum-of-squares (SOS). In the following section, we briefly discuss the application of the moment-based LMI relaxation [16] to Problem (8), which allows us to compute a lower bound of $t^{*}$ (or equivalently, an upper bound on the SSV $\mu_{\Delta}(M)$ ).

\section{LMI RELAXATION: UPPER BOUND ON $\mu_{\Delta}(M)$}

Before discussing the application of the moment-based relaxation [16] to Problem (8), let us introduce some useful definitions.

Let us call the functions defining the set of feasibility of Problem (8) as follows:

$g_{1}=t ; \quad g_{2}=\left\|x_{\mathrm{re}}\right\|_{2}^{2}+\left\|x_{\mathrm{im}}\right\|_{2}^{2}-\bar{x} ;$

$G_{3}=\operatorname{diag}\left(\left(I-M_{\mathrm{re}} \Delta_{\mathrm{re}}+M_{\mathrm{im}} \Delta_{\mathrm{im}}\right) x_{\mathrm{re}}+\left(M_{\mathrm{im}} \Delta_{\mathrm{re}}+M_{\mathrm{re}} \Delta_{\mathrm{im}}\right) x_{\mathrm{im}}\right)$; $G_{4}=\operatorname{diag}\left(\left(I-M_{\mathrm{re}} \Delta_{\mathrm{re}}+M_{\mathrm{im}} \Delta_{\mathrm{im}}\right) x_{\mathrm{im}}-\left(M_{\mathrm{im}} \Delta_{\mathrm{re}}+M_{\mathrm{re}} \Delta_{\mathrm{im}}\right) x_{\mathrm{re}}\right)$; $G_{5}=\left(\begin{array}{cccc}t I & \Delta_{\mathrm{re}} & 0 & -\Delta_{\mathrm{im}} \\ \Delta_{\mathrm{re}}^{\top} & I & \Delta_{\mathrm{im}}^{\top} & 0 \\ 0 & \Delta_{\mathrm{im}} & t I & \Delta_{\mathrm{re}} \\ -\Delta_{\mathrm{im}}^{\top} & 0 & \Delta_{\mathrm{re}}^{\top} & I\end{array}\right) ;$

and let us stack the optimization variables of Problem (8) in a vector $Z \in \mathbb{R}^{n}$, with $n$ being the number of decision variables in $(8)$.

For a sequence $y=\left\{y_{\alpha}\right\}_{\alpha \in \mathcal{A}_{h}^{n}}$ and for a generic polynomial $g \in \mathbb{R}_{h}[Z]$, let us define the map $L_{y}(g)$ as:

$$
g \mapsto L_{y}(g)=\sum_{\alpha \in \mathcal{A}_{h}^{n}} g_{\alpha} y_{\alpha} .
$$

The sequence $y=\left\{y_{\alpha}\right\}_{\alpha \in \mathcal{A}_{h}^{n}}$ is referred as sequence of moments of order $h$ of a (non-specified) Borel probability measure $\nu$ on $\mathbb{R}^{n}$, i.e., $y_{\alpha}=\int Z^{\alpha} \nu(d Z)$. Let us define the so-called moment matrix $N_{h}(y)$ truncated to order $h$ as

$$
N_{h}(y)=L_{y}\left(b_{h}(Z) b_{h}^{\top}(Z)\right),
$$

where the operator $L_{y}$ is applied entry-wise to the matrix $b_{h}(Z) b_{h}^{\top}(Z)$.

Let us define the so-called truncated localizing matrix $N_{h}(g y)$ of order $h$ associated with the polynomial $g$ as:

$$
N_{h}(g y)=L_{y}\left(b_{h}(Z) b_{h}^{\top}(Z) g(Z)\right) .
$$

Similarly, for a matrix $G(Z)$ whose entries are polynomial functions in the variable $Z$, the truncated localizing matrix $N_{h}(G y)$ associated with the polynomial matrix $G$ is defined by:

$$
N_{h}(G y)=L_{y}\left(b_{h}(Z) b_{h}^{\top}(Z) \otimes G(Z)\right) .
$$

For a given integer $h \geq 1$, the SDP-relaxed problem of order $h$ associated to (8) is defined as:

$$
\begin{aligned}
\underline{t}_{h}^{*}= & \min _{\left\{y_{\alpha}\right\}_{\alpha \in \mathcal{A}_{2 h}^{n}}} L_{y}(t) \\
\text { s.t. } & N_{h}(y) \succeq 0, \quad N_{h-1}\left(g_{1} y\right) \succeq 0, \quad N_{h-1}\left(g_{2} y\right) \succeq 0, \\
& N_{h-1}\left(G_{3} y\right)=0, \quad N_{h-1}\left(G_{4} y\right)=0, \quad N_{h-1}\left(G_{5} y\right) \succeq 0 .
\end{aligned}
$$

\section{Theorem 3: The following results hold:}

(i) for any relation order $h \geq 1, \underline{t}_{h}^{*}$ is a lower-bound of $t^{*}$, i.e., $\underline{t}_{h}^{*} \leq t^{*}$;

(ii) $\quad \underline{t}_{h}^{*}$ converges, from below, to $t^{*}$, i.e., $\underline{t}_{h}^{*} \leq \underline{t}_{h+1}^{*} \leq t^{*}$ and $\lim _{h \rightarrow \infty} \underline{t}_{h}^{*}=t^{*}$;

(iii) for any relation order $h \geq 1$, the matrix $I-M \Delta$ is nonsingular for all the uncertainties $\Delta \in \mathcal{B}_{\Delta}^{\sqrt{t_{n}^{*}}}$, or equivalently, $\frac{1}{\sqrt{\underline{t}_{h}^{*}}}$ is an upper bound of $\mu_{\boldsymbol{\Delta}}(M)$. Furthermore:

$$
\mu_{\boldsymbol{\Delta}}(M) \leq \frac{1}{\sqrt{\underline{t}_{h+1}^{*}}} \leq \frac{1}{\sqrt{\underline{t}_{h}^{*}}} ; \lim _{h \rightarrow \infty} \frac{1}{\sqrt{\underline{t}_{h}^{*}}}=\mu_{\boldsymbol{\Delta}}(M) .
$$

Proof: Parts (i) and (ii) follow from a direct application of the moment-based LMI relaxation [16]. A sketch of the proof of part (i) is here provided. Let $Z^{*}$ be the optimizer of the polynomial Problem (8) and let $y^{*}$ be equal to $b_{2 h}\left(Z^{*}\right)$. We remind that $b_{2 h}\left(Z^{*}\right)=\left\{\left(Z^{*}\right)^{\alpha}\right\}_{\alpha \in \mathcal{A}_{2 h}^{n}}$. The point $y^{*}=b_{2 h}\left(Z^{*}\right)$ belongs to the set of feasibility of Problem (13). In fact, $N_{h}\left(y^{*}\right)=b_{h}\left(Z^{*}\right) b_{h}^{\top}\left(Z^{*}\right) \succeq 0$; $N_{h}\left(g_{1} y^{*}\right)=b_{h}\left(Z^{*}\right) b_{h}^{\top}\left(Z^{*}\right) g_{1}\left(Z^{*}\right) \succeq 0$ and $N_{h}\left(g_{2} y^{*}\right)=$ $b_{h}\left(Z^{*}\right) b_{h}^{\top}\left(Z^{*}\right) g_{2}\left(Z^{*}\right) \succeq 0$ since $g_{1}\left(Z^{*}\right) \geq 0$ and $g_{2}\left(Z^{*}\right) \geq 0$; $N_{h}\left(G_{3} y^{*}\right)=b_{h}\left(Z^{*}\right) b_{h}^{\top}\left(Z^{*}\right) \otimes G_{3}\left(Z^{*}\right)=0$ and $N_{h}\left(G_{4} y^{*}\right)=$ $b_{h}\left(Z^{*}\right) b_{h}^{\top}\left(Z^{*}\right) \otimes G_{4}\left(Z^{*}\right)=0$ since $G_{3}\left(Z^{*}\right)=0$ and $G_{4}\left(Z^{*}\right)=0 ; N_{h}\left(G_{5} y^{*}\right)=b_{h}\left(Z^{*}\right) b_{h}^{\top}\left(Z^{*}\right) \otimes G_{5}\left(Z^{*}\right) \succeq 0$ since $G_{5}\left(Z^{*}\right) \succeq 0$. As a consequence, since $L_{y^{*}}(t)=t^{*}$ and, as already discussed, $Z^{*}$ is feasible for Problem (13), then $\underline{t}_{h}^{*} \leq t^{*}$. Part (iii) directly follows from parts (i) and (ii) and Corollary 2 .

The asymptotic convergence properties highlighted in Theorem 3 can be interpreted as follows: the global optimum of the nonconvex optimization Problem (8) can be computed 
by solving a convex SDP problem with infinite-size LMI constrains. Nevertheless, in practice, an accurate (and most of the times exact) approximation of the SSV $\mu_{\Delta}(M)$ is usually attained at a small value of the relaxation order $h$, i.e., $h=2$ (see the examples in Section VI). A discussion on the accuracy of the computed upper bound $\frac{1}{\sqrt{t_{h}^{*}}}$ on $\mu_{\Delta}(M)$ is reported in the next section, together with some techniques that can be used to compute a lower bound on $\mu_{\Delta}(M)$ and a structured matrix $\Delta^{*} \in \Delta$ which makes $I-M \Delta^{*}$ singular.

\section{Computation Of A LOWER BOUnd ON $\mu_{\boldsymbol{\Delta}}(M)$}

Two heuristics to compute a lower bound on $\mu_{\Delta}(M)$, and thus to evaluate the conservativeness of the computed upper bound $\frac{1}{\sqrt{\underline{t}_{h}^{*}}}$, are briefly discussed in this section. The first heuristic is based on well known results of the moment LMI relaxation [15], and it provides a sufficient condition to: (i) verify whether the solution of the SDP-relaxed Problem (13) coincides with exact SSV $\mu_{\boldsymbol{\Delta}}(M)$; (ii) compute a matrix $\Delta^{*} \in \Delta$ which makes $I-M \Delta^{*}$ singular. The second proposed heuristic refines the minimizer of the SDP-relaxed Problem (13) in order to compute, through convex optimization, a matrix $\Delta^{*} \in \Delta$ which renders $I-M \Delta^{*}$ singular.

A. Method 1: Extraction of the global minimizer from the firstorder moments of $y^{*}$

Consider the point $\hat{y}^{*}$ in $\mathbb{R}^{n}$ :

$\hat{y}^{*}:=\left\{y_{\alpha}^{*}: \sum_{i=1}^{n} \alpha_{i}=1, \alpha \in \mathbb{N}_{0}^{n}\right\}=\left\{y_{100 \cdots 00}^{*}, y_{010 \cdots 00}^{*}, \ldots, y_{000 \cdots 01}^{*}\right\}$,

with $y^{*}$ being the minimizer of the SDP-relaxed Problem (13) for a fixed finite value of $h$. It follows that, if $Z^{*}=\hat{y}^{*}$ is a feasible point of the polynomial optimization Problem (8), then $Z^{*}=\hat{y}^{*}$ is also a global minimizer of (8) (see Section 6.6 in [22] for a detailed proof). Thus, $\frac{1}{\sqrt{\underline{t}_{h}^{*}}}$ coincides with $\mu_{\Delta}(M)$ and a structured matrix $\Delta^{*} \in \Delta:\left\|\Delta^{*}\right\|=\sqrt{\underline{t}_{h}^{*}}$ which renders $I-M \Delta^{*}$ singular can be then derived from $Z^{*}$. Based on the author's experience, in most of the cases, the point $\hat{y}^{*}$ has been observed to belong (up to a numerical precision) to the feasible set of Problem (8) for a relaxation order $h \leq 2$. Therefore, in most of the analyzed cases, the SDP-relaxed Problem (13) provides the exact value of the SSV $\mu_{\boldsymbol{\Delta}}(M)$ for $h \leq 2$. It is worth remarking that the use of the first order moments is inspired by the results discussed in [23], where it is shown that, when the moment matrix $N_{h}\left(y^{*}\right)$ has rank one, the first order moment vector $\hat{y}^{*}$ is the global minimizer of the original polynomial optimization problem. In the (rare) cases when $Z^{*}=\hat{y}^{*}$ does not belong to the set of feasibility of Problem (8), the heuristic described in the next subsection can be used to compute a lower bound on $\mu_{\Delta}(M)$.

\section{B. Method 2: Refinement of the first-order moments of $y^{*}$}

In the cases $Z^{*}=\hat{y}^{*}$ in (14) is not feasible for Problem (8) (that is, Method 1 cannot be applied), $Z^{*}=\hat{y}^{*}$ can be used as a starting point to compute a matrix $\Delta \in \Delta$ which makes $I-M \Delta$ singular, as described in the following.
Consider the vector $Z^{*}=\hat{y}^{*}$ and take only the components of $Z^{*}$ associated to the optimization variables $x_{\mathrm{re}}$ and $x_{\mathrm{im}}$. Let us denote such components as $x_{\mathrm{re}}^{*}$ and $x_{\mathrm{im}}^{*}$. Under the condition that the vector $x_{\mathrm{re}}^{*}+j x_{\mathrm{im}}^{*}$ is not null, the key idea of the heuristic described in the following is to find a matrix $\Delta^{*} \in \Delta$ with minimum norm such that $x_{\mathrm{re}}^{*}+j x_{\mathrm{im}}^{*}$ belongs to the null space of the matrix $I-M \Delta^{*}$. Following the same considerations used to derive Problem (8), this idea can be translated into the following convex semidefinite programming problem:

$$
\begin{aligned}
\hat{t}= & \min t \\
& t, \Delta_{\mathrm{re}}, \Delta_{\mathrm{im}} \\
\text { s.t. } & t \geq 0, \\
& \left(I-M_{\mathrm{re}} \Delta_{\mathrm{re}}+M_{\mathrm{im}} \Delta_{\mathrm{im}}\right) x_{\mathrm{re}}^{*}+\left(M_{\mathrm{im}} \Delta_{\mathrm{re}}+M_{\mathrm{re}} \Delta_{\mathrm{im}}\right) x_{\mathrm{im}}^{*}=0, \\
& \left(I-M_{\mathrm{re}} \Delta_{\mathrm{re}}+M_{\mathrm{im}} \Delta_{\mathrm{im}}\right) x_{\mathrm{im}}^{*}-\left(M_{\mathrm{im}} \Delta_{\mathrm{re}}+M_{\mathrm{re}} \Delta_{\mathrm{im}}\right) x_{\mathrm{re}}^{*}=0, \\
& \left(\begin{array}{cccc}
t I & \Delta_{\mathrm{re}} & 0 & -\Delta_{\mathrm{im}} \\
\Delta_{\mathrm{re}}^{\top} & I & \Delta_{\mathrm{im}}^{\top} & 0 \\
0 & \Delta_{\mathrm{im}} & t I & \Delta_{\mathrm{re}} \\
-\Delta_{\mathrm{im}}^{\top} & 0 & \Delta_{\mathrm{re}}^{\top} & I
\end{array}\right) \succeq 0 .
\end{aligned}
$$

The minimizer of (15) thus provides a matrix $\Delta^{*} \in \Delta$, with norm $\left\|\Delta^{*}\right\|=\sqrt{\hat{t}}$, which renders $I-M \Delta^{*}$ singular. A lower bound on the SSV $\mu_{\Delta}(M)$ is then given by $\frac{1}{\sqrt{\hat{t}}}$. Evaluating the level of conservativeness of the described heuristic is not an easy task, nevertheless it provides, in practice, satisfactory results in the (rare) cases when Method 1 fails.

\section{EXAMPLES}

\section{A. Example 1}

Consider the complex matrix $M$ :

$$
M=\left(\begin{array}{rrrrr}
0 & \frac{1}{2} & 1 & 1 & \frac{1}{2} \\
\frac{1}{2} & -\frac{1}{2} & 0 & 0 & \frac{1}{2} \\
0 & 1 & 1 & \frac{1}{2} & 0 \\
-\frac{1}{2} & \frac{1}{2} & -\frac{1}{2} & 1 & \frac{1}{2} \\
\frac{1}{2} & \frac{1}{2} & 0 & -\frac{1}{2} & \frac{1}{2}
\end{array}\right)+i\left(\begin{array}{rrrrr}
1 & -\frac{1}{2} & 0 & 0 & 0 \\
0 & 0 & 1 & 1 & -\frac{1}{2} \\
1 & -\frac{1}{2} & 0 & 0 & 0 \\
0 & 1 & \frac{1}{2} & \frac{1}{2} & -\frac{1}{2} \\
1 & \frac{1}{2} & 0 & -\frac{1}{2} & -\frac{1}{2}
\end{array}\right),
$$

and the block structured uncertainty set $\Delta \subset \mathbb{C}^{5,5}$ :

$$
\boldsymbol{\Delta}=\left\{\boldsymbol{\Delta}=\left(\begin{array}{cccc}
p_{1} & 0 & 0 & 0 \\
0 & p_{1} & 0 & 0 \\
0 & 0 & p_{1} & 0 \\
0 & 0 & 0 & \Delta_{1}
\end{array}\right) \mid p_{1} \in \mathbb{R}, \quad \Delta_{1} \in \mathbb{C}^{2,2}\right\} .
$$

The developed moment-based LMI relaxation has been applied to compute upper and lower bounds on the structured singular value $\mu_{\Delta}(M)$. The Yalmip Matlab interface [24] has been used to construct the SDP-relaxed Problem (13), whose solution has been computed through the solver MOSEK [25]. The obtained bounds are reported in Table I, together with the bounds calculated through the Matlab command mussv, belonging to the Robust Control Toolbox (the reader is referred to [14] for details on the algorithms implemented in the command mussv). The CPU time taken by the solver MOSEK to compute the solution of Problem (13) is 272 seconds on a $2.40-\mathrm{GHz}$ Intel Pentium IV with 3 GB of RAM, while computing bounds on $\mu_{\Delta}(M)$ with the command mussv required 3.2 seconds. Results in Table I show that, the upper and the lower bounds 
TABLE I

EXAMPLE 1. LOWER AND UPPER BOUNDS ON THE SSV $\mu_{\Delta}(M)$ COMPUTED THROUGH THE MATLAB COMMAND musSV AND THROUGH THE MOMENT LMI RELAXATION FOR $\delta=2$.

\begin{tabular}{ccc}
\hline Approach & $\begin{array}{c}\text { Lower bound } \\
\text { on } \mu_{\Delta}(M)\end{array}$ & $\begin{array}{c}\text { Upper bound } \\
\text { on } \mu_{\Delta}(M)\end{array}$ \\
\hline Matlab command mussv & 1.8291 & 2.1100 \\
\hline $\begin{array}{c}\text { Moment LMI } \\
\text { relaxation }\end{array}$ & 2.1007 & 2.1007 \\
\hline
\end{tabular}

on $\mu_{\Delta}(M)$ computed through the Matlab command mussv do not coincide, thus conservativeness is introduced in evaluating $\mu_{\Delta}(M)$. On the other hand, for a relaxation order $\delta=2$, the SDP-relaxed Problem (13) provides the exact value of $\mu_{\boldsymbol{\Delta}}(M)$. This has been verified through the approach discussed in Section V-A, which also provides a structured matrix $\Delta^{*} \in \Delta$ with norm $\left\|\Delta^{*}\right\|=\frac{1}{\sqrt{\underline{t}_{h}^{*}}}=\frac{1}{\sqrt{2.1007}}=0.4760$ which renders $I-M \Delta^{*}$ singular. The numerical value of such a matrix is given by:

$$
\begin{aligned}
& \Delta^{*}=\left(\begin{array}{ccccc}
-0.4760 & 0 & 0 & 0 & 0 \\
0 & -0.4760 & 0 & 0 & 0 \\
0 & 0 & -0.4760 & 0 & 0 \\
0 & 0 & 0 & 0.4511 & -0.1025 \\
0 & 0 & 0 & -0.1025 & -0.1211
\end{array}\right)+ \\
& +i\left(\begin{array}{ccccc}
0 & 0 & 0 & 0 & 0 \\
0 & 0 & 0 & 0 & 0 \\
0 & 0 & 0 & 0 & 0 \\
0 & 0 & 0 & -0.0609 & 0.0910 \\
0 & 0 & 0 & 0.0910 & 0.4354
\end{array}\right) .
\end{aligned}
$$

\section{B. Example 2}

In this example, the SSV is used to analyze the robust stability of linear time-invariant feedback systems under parametric uncertainty. The considered closed-loop configuration is reported in Fig. 1, where the plant $\mathcal{G}$ to be controlled is a mass-damper-spring system whose dynamics are described by the second-order differential equation:

$$
m \ddot{x}(t)+c \dot{x}(t)+k x(t)=u(t),
$$

where $x$ is the displacement of the mass from the equilibrium position, $u$ is an external force acting on the mass, $m \in \mathbb{R}$ is the mass, $c \in \mathbb{R}$ is the damping coefficient and $k \in \mathbb{R}$ is the spring constant. The values of $m, c$ and $k$ are not known exactly, and they are assumed to belong to the (open) uncertainty intervals: $m \in\left(\begin{array}{ll}1.2 & 4.8\end{array}\right), c \in\left(\begin{array}{ll}0.7 & 1.3\end{array}\right), k \in\left(\begin{array}{ll}1.1 & 2.9\end{array}\right)$, or equivalently:

$$
m=m_{\mathrm{o}}+1.8 p_{1}, \quad c=c_{\mathrm{o}}+0.3 p_{2}, \quad k=k_{\mathrm{o}}+0.9 p_{3},
$$

where $m_{\mathrm{O}}=3, c_{\mathrm{o}}=1$ and $k_{\mathrm{O}}=2$ are the nominal values of $m, c$ and $k$, while $p_{1}, p_{2}$ and $p_{3}$ are normalized uncertain parameters which take values within the interval $(-1,1)$. The transfer function of the controller $\mathcal{K}$ is given by:

$$
\mathcal{K}(s)=\frac{-9.0229(s-141.4)\left(s^{2}+0.3333 s+0.6667\right)}{(s+7.997)(s+0.002501)\left(s^{2}+7.263 s+26.54\right)} .
$$

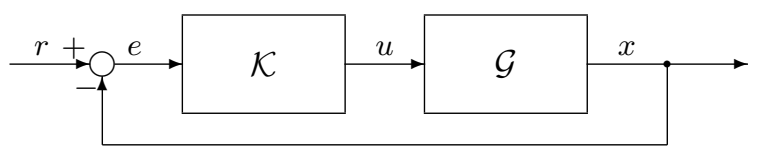

Fig. 1. Feedback control system. $\mathcal{G}$ : plant; $\mathcal{K}$ : controller; $r$ : reference signal; $u$ : external force acting on the mass; $x$ displacement of the mass.
This controller stabilizes the nominal closed-loop system and minimizes the $\mathcal{H}_{\infty}$ norm:

$$
\left\|\begin{array}{c}
\mathcal{W}_{s}\left(1+\mathcal{G}_{\mathrm{o}} \mathcal{K}\right)^{-1} \\
\mathcal{W}_{r} \mathcal{K}\left(1+\mathcal{G}_{\mathrm{o}} \mathcal{K}\right)^{-1}
\end{array}\right\|_{\infty},
$$

with $\mathcal{G}_{\mathrm{o}}$ being the nominal plant, and $\mathcal{W}_{s}$ and $\mathcal{W}_{r}$ being weighting linear filters with transfer functions:

$$
\mathcal{W}_{s}(s)=0.85 \frac{s^{2}+1.8 s+11}{(s+7.997)(s+0.002501)}, \quad \mathcal{W}_{r}(s)=0.01
$$

In order to verify if the controller $\mathcal{K}$ achieves robust stability w.r.t. the considered perturbations on $m, c$ and $k$, a linear fractional representation (LFR) of the considered closed-loop system is derived. The computed LFR has the form:

$$
\begin{aligned}
& {\left[\begin{array}{c}
z_{\Delta} \\
z
\end{array}\right]=\left[\begin{array}{ll}
N_{11} & N_{12} \\
N_{21} & N_{22}
\end{array}\right]\left[\begin{array}{c}
w_{\Delta} \\
r
\end{array}\right], z=\left[\begin{array}{l}
e \\
u
\end{array}\right],} \\
& z_{\Delta}=\underbrace{\left(\begin{array}{ccc}
p_{1} & 0 & 0 \\
0 & p_{2} & 0 \\
0 & 0 & p_{3}
\end{array}\right)}_{\Delta} w_{\Delta},
\end{aligned}
$$

where $N_{11}, N_{12}, N_{21}$ and $N_{22}$ are known LTI systems, whose transfer functions $N_{11}(s), N_{12}(s), N_{21}(s)$ and $N_{22}(s)$ can be computed through block diagram manipulations. According to the SSV theory (see, e.g., [1]), the feedback system in Fig. 1 is guaranteed to be stable for all values of $p_{1}, p_{2}, p_{3} \in(-1,1)$ if and only if:

$$
\mu_{\Delta}\left(N_{11}(i \omega)\right) \leq 1 \quad \text { for all } \omega \in \mathbb{R} \cup\{\infty\} .
$$

The lower and upper bounds on $\mu_{\boldsymbol{\Delta}}\left(N_{11}(i \omega)\right)$ computed through the Robust Control Toolbox are plotted in Fig. 2 over a set of 1000 frequency points $\omega$ logarithmically equally spaced in the interval $[0.1100]$. For each $\omega$, the average CPU time taken to compute lower and upper bounds on $\mu_{\Delta}\left(N_{11}(i \omega)\right)$ is 0.12 seconds. Note that, based on the results in Fig. 2, we can claim neither that the feedback system is robustly stable (since there exists at least a value of $\omega$ such that the computed upper bound on $\mu_{\boldsymbol{\Delta}}\left(N_{11}(i \omega)\right)$ is greater than 1$)$ nor that there exist some values of $p_{1}, p_{2}, p_{3} \in(-1,1)$ which destabilize the closed-loop system (in fact, the computed lower bound on $\mu_{\Delta}\left(N_{11}(i \omega)\right)$ is always smaller than 1 within the considered frequency range). Lower and upper bounds on $\mu_{\boldsymbol{\Delta}}\left(N_{11}(i \omega)\right)$ are now computed through the moment LMI relaxation discussed in the paper, by solving the SDP Problem (13) for a relaxation order $\delta=2$. For each $\omega$, the average CPU time taken by solver MOSEK to compute the solution of Problem (13) is 13 seconds. The obtained bounds are plotted in Fig. 3, which shows that, in most of the considered frequency points $\omega$, upper and lower bounds coincide, thus the exact value of $\mu_{\boldsymbol{\Delta}}\left(N_{11}(i \omega)\right)$ is achieved. The only frequency points $\omega$ where lower and upper bounds do not coincide are in the range $\left[\begin{array}{ll}0.265 & 0.331\end{array}\right] \mathrm{rad} / \mathrm{s}$ and at $\omega=13.55 \mathrm{rad} / \mathrm{s}$. Within the range $\left[\begin{array}{ll}0.265 & 0.331\end{array}\right] \mathrm{rad} / \mathrm{s}$, the computed lower bound on $\mu_{\boldsymbol{\Delta}}\left(N_{11}(i \omega)\right)$ is equal to 0 (i.e., no matrix $\Delta$ which makes $I-N_{11}(i \omega) \Delta$ singular has been found). At $\omega=13.55$ $\mathrm{rad} / \mathrm{s}$, the gap between the upper and lower bound (computed through the heuristic described in Section V-B) is 0.0475 . Note that, from Fig. 3, we can claim that there exist values of 


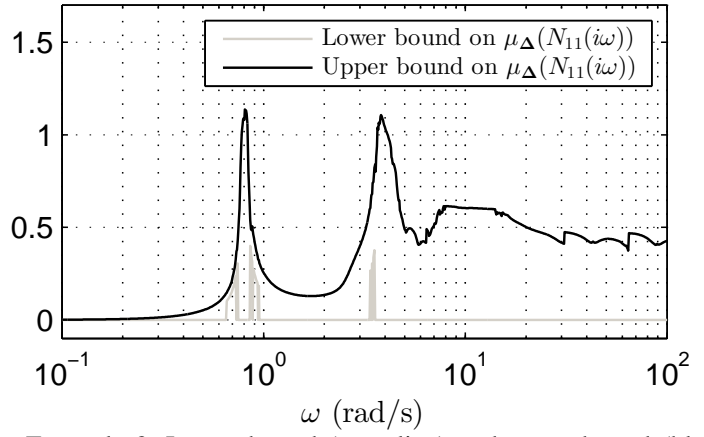

Fig. 2. Example 2. Lower bound (gray line) and upper bound (black line) on the structured singular value $\mu_{\boldsymbol{\Delta}}\left(N_{11}(i \omega)\right)$ computed with the Robust Control Toolbox in Matlab.

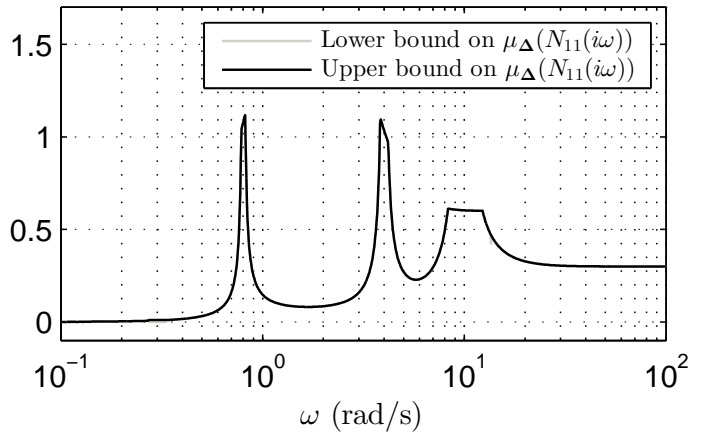

Fig. 3. Example 2. Lower bound (gray line) and upper bound (black line) on the structured singular value $\mu_{\boldsymbol{\Delta}}\left(N_{11}(i \omega)\right)$ computed through the moment LMI relaxation. The gray and the black lines are overlapped, except in the frequency range $\left[\begin{array}{ll}0.265 & 0.331\end{array}\right] \mathrm{rad} / \mathrm{s}$ and at $\omega=13.55 \mathrm{rad} / \mathrm{s}$.

$p_{1}, p_{2}, p_{3} \in(-1,1)$ destabilizing the closed-loop system. In fact, $\mu_{\boldsymbol{\Delta}}\left(N_{11}(i \omega)\right)$ is greater than one at some frequencies. For example, for $\omega_{\mathrm{o}}=0.8182 \mathrm{rad} / \mathrm{s}, \mu_{\Delta}\left(N_{11}\left(i \omega_{\mathrm{o}}\right)\right)=1.1178$. The values of the uncertain parameters $p_{1}, p_{2}$ and $p_{3}$ leading to $\mu_{\boldsymbol{\Delta}}\left(N_{11}\left(i \omega_{\mathrm{o}}\right)\right)=1.1178$, and destabilizing the closed-loop system, are equal to: $p_{1}=-0.7815, p_{2}=-0.8945$ and $p_{3}=0.8946$. These values have been computed through the method discussed in Section V-A.

\section{CONCLUSIONS}

A new approach to compute an upper bound on the Structured Singular Value (SSV) of a matrix w.r.t. a set of structured mixed real/complex uncertainties is presented in this paper. The main idea underlying the method is to formulate the computation of the SSV as a polynomial optimization problem which is solved through efficient moment-based LMI relaxations. Two heuristics to compute lower bounds on the SSV are also presented. The reported numerical examples show that the developed method provides significantly tighter bounds compared to the ones computed with the algorithms implemented in the Robust Control Toolbox in Matlab, and, in most of the cases, it provides the exact value of the SSV. The presented algorithm can efficiently handle, in commercial workstations, uncertain matrices with about 7 different real parametric uncertainties or with about 5 different complex uncertainties. Furthermore, it is worth pointing out that the computational load of the proposed algorithm decreases when "highly structured" uncertainties (e.g., uncertainties with real repeated blocks) are considered. In fact, in this case, the formulated polynomial optimization problem involves a lower number of decision variables. Ongoing activities are devoted to extending the ideas underlying the developed method for the design of robust controllers.

\section{ACKNOWLEDGMENT}

The author is grateful to Dr. Roland Tóth, Prof. Carsten Scherer, Prof. Graziano Chesi, Mr. Bruno Londino, to the Associate Editor and to the anonymous Reviewers for their valuable comments to improve the contribution of the paper.

\section{REFERENCES}

[1] J. C. Doyle, "Analysis of feedback systems with structured uncertainties," in IEE Proceedings D (Control Theory and Applications), vol. 129, no. 6, 1982, pp. 242-250.

[2] S. Polijak and J. Rohn, "Checking robust non-singularity is NP-hard," Mathematics of Control, Signals, and Systems, vol. 6, no. 2, pp. 1-9, 1993.

[3] R. P. Braatz, P. M. Young, J. C. Doyle, and M. Morari, "Computational complexity of $\mu$ calculation," IEEE Trans. on Automatic Control, vol. 39, no. 5, pp. 1000-1002, 1994.

[4] M. K. H. Fan and A. L. Tits, "Characterization and efficient computation of the structured singular value," IEEE Trans. on Automatic Control, vol. 31, no. 8, pp. 734-743, 1986.

[5] P. M. Young and J. C. Doyle, "Computation of $\mu$ with real and complex uncertainties," in Proc. of the 29th IEEE Conference on Decision and Control, 1990, pp. 1230-1235.

[6] M. K. H. Fan, A. L. Tits, and J. C. Doyle, "Robustness in the presence of mixed parametric uncertainty and unmodeled dynamics," IEEE Trans. on Automatic Control, vol. 36, no. 1, pp. 25-38, 1991.

[7] V. Balakrishnan, E. Feron, S. Boyd, and L. El Ghaoui, "Computing bounds for the structured singular value via an interior point algorithm," in Proc. of the American Control Conference, 1992, pp. 2195-2196.

[8] A. Packard and J. C. Doyle, "The complex structured singular value," Automatica, vol. 29, no. 1, pp. 71-109, 1993.

[9] G. Ferreres, A practical approach to robustness analysis. Springer, 1999.

[10] G. Ferreres, J. Magni, and J. Biannic, "Robustness analysis of flexible structures: practical algorithms," International Journal of Robust and Nonlinear Control, vol. 13, no. 8, pp. 715-733, 2003.

[11] P. M. Young and J. C. Doyle, "A lower bound for the mixed $\mu$ problem," IEEE Trans. on Automatic Control, vol. 42, no. 1, pp. 123-128, 1997.

[12] C. W. Scherer, "Lmi relaxations in robust control," European Journal of Control, vol. 12, no. 1, pp. 3-29, 2006.

[13] M. Karow, E. Kokiopoulou, and D. Kressner, "On the computation of structured singular values and pseudospectra," Systems \& Control Letters, vol. 59, no. 2, pp. 122-129, 2010.

[14] G. Balas, R. Chiang, A. Packard, and M. Safonov, "Robust control toolbox 3," 2005.

[15] J. B. Lasserre, "Global optimization with polynomials and the problem of moments," SIAM J. on Optimization, vol. 11, pp. 796-817, 2001.

[16] D. Henrion and J. B. Lasserre, "Convergent relaxations of polynomial matrix inqequalities and static output feedback," IEEE Trans. on Automatic Control, vol. 51, no. 2, pp. 192-202, 2006.

[17] — "Solving nonconvex optimization problems," IEEE Control Systems Magazine, vol. 24, no. 3, pp. 72-83, 2004.

[18] J. Nie, "Optimality conditions and finite convergence of Lasserre's hierarchy," Mathematical programming, vol. 146, no. 1-2, pp. 97-121, 2014.

[19] V. Cerone, D. Piga, and D. Regruto, "Robust pole placement for plants with semialgebraic parametric uncertainty," in Proc. of the American Control Conference, Montréal, Canada, 2012, pp. 5240-5245.

[20] G. Chesi, A. Garulli, A. Tesi, and A. Vicino, "Solving quadratic distance problems: an LMI-based approach," IEEE Transactions on Automatic Control, vol. 48, no. 2, pp. 200-212, 2003.

[21] P. Parrillo, "Semidefinite programming relaxations for semialgebraic problems," Mathematical Programming, vol. 96, pp. 293-320, 2003.

[22] M. Laurent, "Sums of squares, moment matrices and optimization over polynomials," Emerging Applications of Algebraic Geometry, Vol. 149 of IMA Volumes in Mathematics and its Applications, M. Putinar and S. Sullivant (eds.), pp. 157-270, 2009.

[23] D. Henrion and J. B. Lasserre, "Detecting global optimality and extracting solutions in gloptipoly," in Positive polynomials in control, D. Henrion and A. Garulli (eds.). Springer, pp: 293-310, 2005.

[24] J. Löfberg, "YALMIP: A toolbox for modeling and optimization in Matlab," in IEEE International Symposium on Computer Aided Control Systems Design, Taipei, Taiwan, 2004, pp. 284-289.

[25] E. D. Andersen, C. Roos, and T. Terlaky, "On implementing a primaldual interior-point method for conic quadratic optimization," Mathematical Programming, vol. 95, no. 2, pp. 249-277, 2003. 